\title{
La regulación industrial y la transferencia internacional de tecnología en España (1959-1973)
}

\author{
Industrial regulation and technology transfer in Spain (1959-1973)
}

\author{
MAR CEBRIÁN VILLAR \\ Universidad de Salamanca
}

\begin{abstract}
RESUMEN
En este trabajo se estudia la relación entre el cambio tecnológico, el tamaño empresarial y la concentración del mercado en el caso concreto de España en el período 1959-1973. A pesar de la abundante bibliografía, aún no existe una respuesta unánime sobre la relación entre la estructura del mercado y la innovación. Los resultados que aquí se presentan apoyan la hipótesis de Schumpeter que afirma que la innovación aumenta con la concentración del

mercado. Existen, también, otras variables, como el grado de diferenciación del producto, que también afectan a la actividad innovadora.
\end{abstract}

PALABRAS CLAVE: Tamaño empresarial, Estructura del mercado, Cambio tecnológico, Hipótesis de Schumpeter Códigos JEL: O33, 038, N44, L50

\section{ABSTRACT}

This work analyses the relationship between technological change, firm size and market structure, applied to the Spanish economy during 1959-1973. Despite the voluminous literature, the theory yields ambiguous predictions about the effect of market concentration on innovative activity. My results support Schumpeter's hypothesis that innovation increases with market concentration. This empirical work has also found that other variables, such as the extent of product differentiation, can affect innovative activity.

KEY WORDS: Size firm, Market structure, Technological change, Schumpeter's hypothesis

JEL Codes: O33, 038, N44, L50 


\section{Introducción ${ }^{1}$}

E 1 propósito de este artículo es conocer la influencia que el marco institucional pudo tener en la adopción de nuevas tecnologías en España en el período 1959-1973, el de máximo crecimiento de toda su historia. Una de las principales características de dicho marco institucional fue el elevado grado de intervencionismo y las numerosas trabas a la libre competencia ${ }^{2}$. A su vez, la compra de tecnología extranjera fue un elemento clave de la política económica del gobierno franquista como forma de acceder a las innovaciones técnicas ${ }^{3}$. Así, por ejemplo, España destinaba solamente un 0,3 por 100 a I+D en el año 1971 frente al 2,6, 2,1, o 1,8 por 100, de EE.UU., Alemania o Francia, respectivamente. Además, las empresas españolas empleaban pocos recursos en investigación básica, siendo mucho más importantes la investigación aplicada y el desarrollo tecnológico. En 1967 las empresas españolas simplemente destinaban un 5,37 por 100 a la investigación básica, frente a un 36,44 por 100 para la investigación aplicada y un 58,19 por 100 para el desarrollo tecnológico. En 1973 tales cifras eran 3,02, 30,7 y 66,21 por 100, respectivamente.

Al mismo tiempo, el país gastaba más en la compra de tecnología extranjera que en la generación de tecnología propia, destinándose un 0,29 por 100 del PIB en 1964 a la adquisición de tecnología extranjera frente a un 0,14 por 100 para los gastos en I+D. En 1973 estas cifras eran del 0,4 y 0,31 por 100. Otro indicador que refleja la importancia de la tecnología extranjera es la relación de dependencia tecnológica, esto es, el cociente entre los gastos españoles en royalties por patentes, licencias y copyright, y los ingresos que España obtenía por la venta de su propia tecnología. Si en 1963 la dependencia tecnológica de la economía española era de 2,8, diez años más tarde, dicha cifra era de 15,6, es decir, se había multiplicado por más de cinco. Estos números son mucho más elevados que los obtenidos para otros países, como

1 Para la elaboración de este artículo he contado con la generosidad de Santiago López, Giovanni Federico, Bronwyn Hall, Joan Rosès, Julio Tascón, Jesús María Valdaliso y Antonio Navas. Agradezco, asimismo, a los tres evaluadores anónimos de Investigaciones de Historia Económica los comentarios realizados, al personal del Archivo General de la Administración su atención, y al Instituto Universitario Europeo por la organización de un seminario para la discusión de una primera versión de este trabajo. Los errores que el lector pueda encontrar son de mi exclusiva responsabilidad.

2 Comín (1997) ha destacado, como uno de los principales factores que limitaron el crecimiento español en el período 1959-1973, la insuficiente liberalización de la economía, el excesivo intervencionismo y la incapacidad para adoptar las tecnologías más avanzadas. Sobre el alto grado de intervencionismo de la economía española, véase además Fraile (1991).

3 La escasa importancia que siempre ha tenido la inversión en I+D en el crecimiento económico español está documentada en Martín González y Rodríguez (1979), López (1992), Sáiz (1999), Molero y Buesa (1995), Sanz y López (1997), Maluquer de Motes (2000), López y Valdaliso (2001) y Comín y Gálvez (2003). Las carencias de la investigación científica y técnica fueron uno de los factores que limitaron las exportaciones españolas en el período del primer franquismo (Barciela, 2002, p. 364). 
Francia (2,2 y 3,3 en 1963 y 1972, respectivamente), Holanda (1,1 y 1,5), Japón (16,5 y 7,7) o Italia (3,5 en 1965 y 5,6 en 1972) $)^{4}$.

En las páginas que siguen se ofrece nueva información con el objetivo de analizar la relación entre los gastos tecnológicos, la política industrial, el tamaño de las empresas y la estructura del mercado en un país en vías de desarrollo en términos internacionales, como era España en el período 1959-1973. La estructura del mercado es relevante pues refleja características importantes de la situación empresarial entre las que cabe citar las barreras a la entrada y el número y la distribución del tamaño de las empresas ${ }^{5}$. Todas estas características influyen en la rivalidad del mercado y ésta determina, a su vez, las oportunidades económicas y las restricciones a las que las empresas hacen frente cuando planean invertir en nuevas tecnologías. Un hecho bien conocido por todos es que durante el régimen franquista la política española privilegió a ciertos grupos de presión, otorgándoles incentivos que les permitían alcanzar un elevado poder de mercado. Por lo tanto, una de las características del régimen de Franco fue un alto grado de intervencionismo y regulación de la economía ${ }^{6}$. La política que se aplicó después del importante cambio que supuso el Plan de Estabilización del año 1959 continuó, sin embargo, favoreciendo los comportamientos monopolísticos ${ }^{7}$. El alto nivel de regulación de la industria española incrementó las barreras legales de entrada a las nuevas empresas, erosionando así la competencia. Además, a la debilidad de la política a favor de la competencia se unió un alto nivel de protección del mercado español hasta la entrada en la Comunidad Económica Europea en $1986^{8}$.

Un aspecto que atañe muy directamente a las estructuras oligopolísticas y monopolísticas del mercado español fue la regulación industrial, dado que ésta establecía barreras de entrada a los nuevos competidores y favorecía a los ya establecidos, garantizándoles unas mayores cuotas de mercado ${ }^{9}$. Tener una cuota de mercado asegurada podía reducir los incentivos para que las empresas más reguladas adoptasen nuevas tecnologías. Por otra parte, y debido al reducido número de empresas de los mercados altamente regulados, los beneficios de adoptar nuevas

4 Estos datos se derivan de la información suministrada por la balanza de pagos que ofrece el Fondo Monetario Internacional (Cebrián, 2004, epígrafe 1.3).

$5 \quad$ Véanse Dosi (1988), Tirole (1988), capítulo 1, y Cohen y Levin (1989).

$6 \quad$ Para saber más sobre la regulación industrial, véanse Fraile (1999), Valdaliso (2004) y Pires (2003).

7 González (1979), p. 317. Para conocer más la política intervencionista del régimen franquista, véanse Buesa (1982), Martín Aceña y Comín (1991), Catalán (1995), Barciela, López, Melgarejo y Miranda (2001), Serrano Sanz y Pardos (2002) y Barciela (2002).

8 Véanse Steinkamp y García Viñuela (1977), Roldán, Muñoz y Serrano (1978), Viñas, Viñuela, Eguidazu, Fernández y Florensa (1979), Alonso (1990), Serrano Sanz (1997) y Barciela, López, Melgarejo y Miranda (2001).

9 Buesa y Pires (2002). 
tecnologías se incrementan ${ }^{10}$. Ante esta disyuntiva, el objetivo es saber cuál de las dos opciones predominó en el caso de las empresas con un mayor grado de regulación industrial (esto es, bajo el sistema administrativo del régimen franquista de autorización previa necesaria para el establecimiento, ampliación y traslado de plantas fabriles) y cuál para las empresas más liberalizadas. Dicho sistema fue la política más significativa de la regulación industrial en España en el período estudiado. El sistema fue liberalizado en 1963, pero el nivel de regulación se incrementó a partir de $1967^{11}$. La inclusión de los sectores que requerían autorización administrativa previa después de 1967 dependió de la presión que ejercieron los distintos grupos sobre el Gobierno a la hora de pedir mayor protección frente a los nuevos competidores. Los sectores más regulados eran, además, aquellos donde predominaban las empresas de mayor tamaño, con mayores tasas de crecimiento y con una estructura de mercado oligopolística ${ }^{12}$. Se puede concluir afirmando que la regulación industrial en España fue un claro determinante del nivel de concentración empresarial ${ }^{13}$. ¿Cómo influyó la política industrial en la estructura del mercado y en la transferencia tecnológica? ¿Son las relaciones entre una alta regulación industrial (mayor concentración), un mayor tamaño empresarial y mayores gastos en la adquisición de nuevas tecnologías estadísticamente significativas?

No hay muchos estudios que hayan analizado la relación entre el tamaño empresarial, la concentración de los mercados y la inversión en nuevas tecnologías en los países relativamente más atrasados. Algunas excepciones son los trabajos de Braga y Willmore (1991) y Siddharthan y Agarwal (1992). En ambas investigaciones se concluye que, en los casos de Brasil e India respectivamente, existe una relación positiva entre el tamaño empresarial y la compra de tecnología extranjera. Sin embargo, tras incluir más variables en el análisis, los autores encuentran que el tamaño de la empresa no es una variable importante para determinar los gastos empresariales en la compra de tecnología extranjera ${ }^{14}$. El presente artículo concluye que el gasto en nuevas tecnologías aumenta cuanto más concentrado está el mercado. Pero también otros factores, como el tipo de bienes producidos por una empresa, se tornan relevantes para explicar el gasto empresarial en el cambio tecnológico.

Dado que las empresas instaladas en España, como se ha visto anteriormente, gastaron muy poco en investigación y desarrollo y dependieron relativamente más

\footnotetext{
$10 \quad$ Hall y Khan (2003).

11 El 10 por 100 de la producción efectiva se encontraba bajo el régimen de autorización previa para el establecimiento y expansión de plantas fabriles. Dicho porcentaje era del 60 por 100 en el período 1968-1973 (Buesa y Pires, 2002, p. 165).

12 Buesa y Pires (2002), pp. 175 y 183.

13 Viscusi, Vernon y Harrington (1995), p. 61.

14 Siddharthan (1992).
} 
que en otros países de la tecnología importada, las inversiones relacionadas con la importación de tecnología son mucho más importantes y dominan los gastos en nuevas tecnologías. Por lo tanto, en el presente trabajo se han considerado los gastos de las empresas españolas en la compra de nuevas tecnologías por medio de los contratos de transferencia de tecnología extranjera como una medida de la innovación ${ }^{15}$. Esto es una limitación del trabajo, puesto que deja fuera los gastos en I+D de las empresas españolas, así como parte de los gastos en la adquisición de las tecnologías incorporadas en los bienes de equipo. Es cierto que esta medida no es completa, pero creo que es la más importante en un país como España que ha progresado gracias a la imitación de tecnologías extranjeras, y donde la generación propia de innovaciones técnicas a partir de actividades de $\mathrm{I}+\mathrm{D}$ era casi inexistente.

Otro supuesto del trabajo es que las cantidades que se gastaron en las tecnologías transferidas por un contrato de asistencia técnica eran muy superiores a las que se pagaban por los contratos de licencia ${ }^{16}$. He calculado que la cantidad media pagada por un contrato de asistencia técnica era más de 40 millones de pesetas y sólo 1,5 millones de pesetas anuales en el caso concreto de los contratos de licencia ${ }^{17}$. Considerando que el 70 por 100 de los contratos que ofrecen información tiene una duración de 5 años, es evidente que la cantidad pagada por un contrato de licencia es muy inferior a la que se deriva de un contrato de asistencia técnica ${ }^{18}$.

15 El Manual de Oslo considera como una innovación la introducción de nuevos productos y procesos en el mercado. Este manual define innovación como cualquier gasto realizado que contribuya a la innovación de un producto o un proceso. Ello engloba la maquinaria y la llamada "tecnología inmaterial" (el software y los materiales de información para poder innovar). Como han señalado Archibugi, Cesaratto y Sirilli (1991), Napolitano (1991) y Calvo (2000), en la medida en que los gastos en I+D sean solamente una parte del total de los gastos asociados al proceso completo de innovación, entonces si las actividades innovadoras se aproximan mediante los gastos en $\mathrm{I}+\mathrm{D}$ se incurre en una infravaloración de los mismos, sobre todo en el caso de las empresas pequeñas y de los sectores tradicionales. Esto es especialmente cierto para las empresas con menos de 200 empleados, es decir, la mayoría de las empresas españolas en el período aquí estudiado, donde los gastos en I+D no son muy relevantes, y para un país que ha primado la compra de tecnología extranjera por encima de la inversión en I+D.

16 Podemos distinguir dos tipos de contratos de licencia: aquéllos que solamente atañen a la licencia para el uso de una tecnología extranjera, y los que, además, incluyen la asistencia técnica (que podía consistir en el envío de personal extranjero a la empresa española o sólo de manuales o dibujos). Si a estos dos añadimos los que eran exclusivamente de asistencia técnica, podemos concluir que hay tres clases de contratos de transferencia de tecnología: los que sólo conceden una licencia; los que incluyen una licencia más la asistencia técnica (ambos se han incluido en la categoría contratos de licencia en el presente trabajo), y los que sólo consisten en la asistencia técnica extranjera.

17 La información sobre la cantidad total pagada al licenciador se ha obtenido a partir de un total de 256 contratos de asistencia técnica y los royalties anuales a partir de 140 contratos de licencia. No se han utilizado todos los contratos porque los pagos totales se desconocen en la mayoría de los casos; muchos contratos recogen el canon anual (un porcentaje sobre el precio de venta, por ejemplo) pero, al ignorarse la producción o las ventas de la empresa, no puede reconstruirse el total pagado. 
La base de datos utilizada engloba un total de 1.504 contratos de transferencia de tecnología (925 contratos de licencia y 579 contratos de asistencia técnica) que fueron firmados entre empresas multinacionales y empresas residentes en España ${ }^{19}$. La información se encuentra en el Archivo General de la Administración (AGA, en adelante) de Alcalá de Henares. Aunque la base de datos incluye contratos desde el año 1928, el grueso se refiere a los años 1964-1972. Se ha leído cada uno de los contratos, con una media de 20 hojas por contrato. La información recogida en los contratos hace referencia, principalmente, al proceso de negociaciones entre las partes, tipo de tecnología transferida, duración del contrato, cláusulas del mismo, nombre y domicilio social del licenciador y del licenciado, año de la firma y año de aprobación del contrato, pagos derivados de éste, forma de pago, justificación de la firma y mercado de venta de la tecnología transferida ${ }^{20}$.

El resto del artículo está estructurado en cinco apartados. En el número dos, se describen las principales pautas de la compra de tecnología extranjera de la economía española. El tercero se centra en los aspectos teóricos sobre regulación, tamaño empresarial y adopción de nuevas tecnologías. Las variables y las principales hipótesis se presentan en el cuarto. El quinto ofrece el modelo empírico y la interpretación de los principales resultados. Finalmente, el último contiene un resumen de las principales conclusiones del artículo.

\section{Las pautas de la transferencia de tecnología en España}

En este aparatado se describen y explican las principales pautas de adquisición de tecnología extranjera en España en función del tipo de contrato, de la tecnología transferida y del tamaño de las empresas compradoras de tecnología. A tal efecto se han elaborado el Cuadro 1 y los Gráficos 1 y 2.

Básicamente, los bienes de consumo (93,8 por 100$)$, bienes intermedios (83,9 por $100)$ y bienes de equipo $(85,5$ por 100$)$ producidos con tecnología extranjera importada por vía contractual, eran fabricados por medio de un contrato de licencia. Por lo tanto, el objetivo fundamental de los contratos de licencia era la fabricación en España de nuevos productos (Gráfico 1).

19 El número de contratos firmados entre 1964 y 1972, según la revista Economía Industrial, estaría en torno a los 3.500 y mi base incluye para esos mismos años 1.212 contratos (34,62 por 100 del total).

20 El AGA no tiene todos los contratos de transferencia tecnológica que fueron firmados por las empresas españolas. Sin embargo, no existen indicios que lleven a pensar que la muestra es sesgada. Además, he podido comprobar que los contratos están archivados en cajas de manera totalmente aleatoria en lo que se refiere a los distintos fondos, años, sectores y empresas. 


\section{GRÁFICO 1}

DESCRIPCIÓN DE LOS PRINCIPALES CONTRATOS DE LICENCIA, 1928-1973

(Número de contratos)

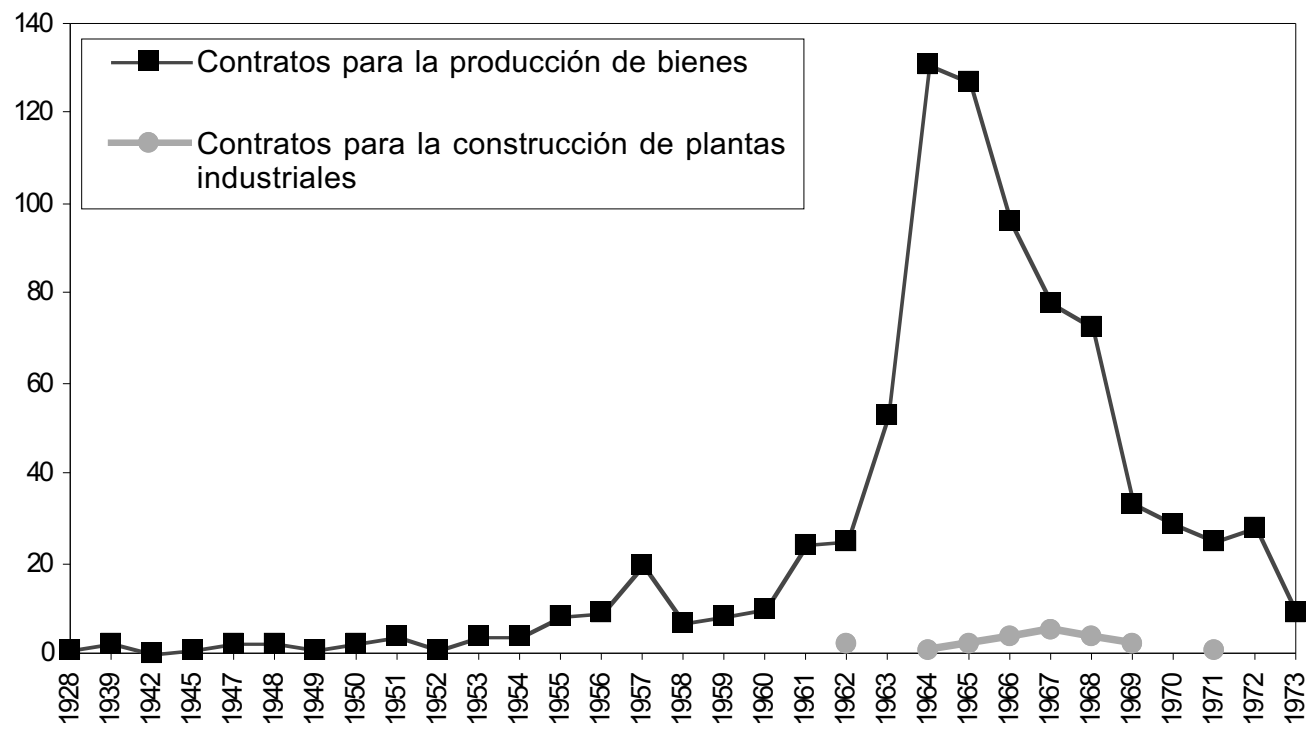

Fuentes: Véase la información proporcionada en el texto.

En cambio, y tal como muestra el Gráfico 2 , los contratos de asistencia técnica tenían como finalidad principal la construcción, total o parcial de nuevas plantas industriales para la fabricación en masa (94,1 por 100).

La razón que explica por qué normalmente se elegía un contrato de asistencia técnica cuando se trataba de construir una nueva planta era la complejidad de la tecnología que se adquiría. Veamos el siguiente ejemplo. En la situación 1, un empresario tiene una empresa para producir ropa y firma un contrato de licencia con el objetivo de ampliar la producción a nuevos diseños. En la situación 2, en cambio, nos encontramos con un empresario que quiere instalar una nueva empresa para el refinado de petróleo. En el primero de estos dos escenarios, no son necesarias nuevas máquinas, dado que la planta está lista para la producción de los nuevos modelos de ropa. Sin embargo, en la segunda situación, se requiere un enorme esfuerzo de inversión, así como la compra de nuevo equipo. Todo en este último caso es nuevo: la forma de producir, el proceso productivo, etc. El know-how que requiere la instalación de esta planta de refinado es muy diferente al que necesita el empresario que quiere 


\section{GRÁFICO 2}

DESCRIPCIÓN DE LOS PRINCIPALES CONTRATOS DE ASISTENCIA TÉCNICA

(Número de contratos)

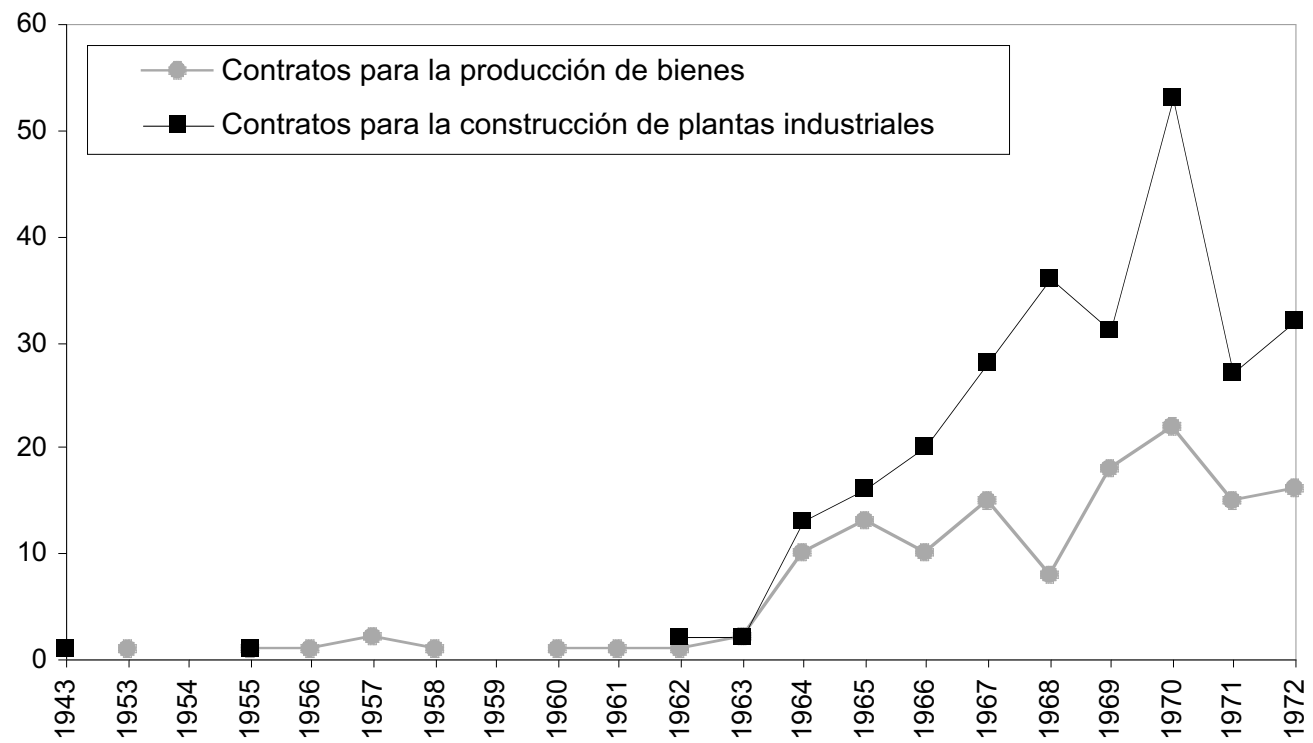

Fuentes: Véase la información proporcionada en el texto.

ampliar su negocio para nuevos diseños de ropa. Una nueva instalación requiere una tecnología mucho más compleja y unos gastos mayores en tecnología y maquinaria, tal y como muestran los datos empíricos deducidos de los contratos estudiados.

Como se observa en el Cuadro 1, la mayoría de los contratos de transferencia de tecnología tenían como objetivo la producción de bienes y la construcción o ampliación de plantas industriales. Los bienes de producción son el principal objeto de los contratos de licencia ${ }^{21}$. Las técnicas transferidas por medio de los contratos de licencia eran, generalmente, como muestra la base de datos, muy estandarizadas y el conocimiento científico que se requería para su producción podía extraerse de los

21 Entre los principales bienes de consumo fabricados mediante un contrato de licencia cabe citar: champú, chicle, productos cosméticos, vestidos, trajes, zumo de naranja, productos de panadería, sopas, salsas, bicicletas, manufacturas metálicas (frigoríficos, calentadores domésticos, lavadoras, máquinas de coser, aspiradores, televisores, tocadiscos), zapatos, relojes, joyas, manufacturas de madera y juguetes. 


\section{CUADRO 1}

OBJETIVOS DE LOS CONTRATOS DE TRANSFERENCIA DE TECNOLOGÍA

\begin{tabular}{|c|c|c|c|c|c|c|c|c|}
\hline & $\begin{array}{l}\text { Bienes de } \\
\text { consumo }\end{array}$ & $\begin{array}{l}\text { Bienes de } \\
\text { producción }\end{array}$ & $\begin{array}{c}\text { Bienes } \\
\text { intermedios }\end{array}$ & $\begin{array}{l}\text { Nueva } \\
\text { planta }\end{array}$ & Estudio & $\begin{array}{l}\text { Reparación } \\
\text { maquinaria }\end{array}$ & Otros & Total \\
\hline \multicolumn{9}{|c|}{$\begin{array}{l}\text { Contratos de } \\
\text { Licencia }\end{array}$} \\
\hline (a) & 227 & 465 & 209 & 18 & 0 & 0 & 6 & 925 \\
\hline (b) & 24,54 & 50,27 & 22,59 & 1,95 & 0,00 & 0,00 & 0,65 & 100 \\
\hline (c) & 93,80 & 85,48 & 83,94 & 5,88 & 0,00 & 0,00 & 75,00 & \\
\hline \multicolumn{9}{|c|}{$\begin{array}{l}\text { Contratos de } \\
\text { Asistencia } \\
\text { Técnica }\end{array}$} \\
\hline (d) & 15 & 79 & 40 & 288 & 117 & 38 & 2 & 579 \\
\hline (e) & 2,59 & 13,64 & 6,91 & 49,74 & 20,21 & 6,56 & 0,35 & 100 \\
\hline (f) & 6,20 & 14,52 & 16,06 & 94,12 & 100,00 & 100,00 & 25,00 & \\
\hline Total & 242 & 544 & 249 & 306 & 117 & 38 & 8 & 1.504 \\
\hline (g) & 16,09 & 36,17 & 16,56 & 20,35 & 7,78 & 2,52 & 0,53 & 100 \\
\hline
\end{tabular}

Notas:
(a) Número de contratos.
(b) Porcentaje sobre el total de contratos de licencia.
(c) Porcentaje sobre el total correspondiente.
(d) Número de contratos.
(e) Porcentaje sobre el total de contratos de asistencia técnica.
(f) Porcentaje sobre el total correspondiente.
(g) Porcentaje sobre el total de contratos.
Entre los contratos cuya finalidad era realizar un estudio es muy común encontrar estudios geológicos, redacción de pro- yectos para futuras posibles inversiones y estudios químicos.
Fuentes: Véase la información proporcionada en el texto.

propios manuales. En el caso de España, sin embargo, la asistencia técnica extranjera fue necesaria, dado el relativo bajo nivel tecnológico de los trabajadores y la falta de ingenieros con los suficientes conocimientos y práctica sobre las formas de organizar la producción. Las únicas barreras a la transferencia de tecnología eran las cantidades que había que pagar por su adquisición, pagos que en el caso de los contratos de licencia no eran muy elevados. Una gran proporción de las empresas españolas que compraron tecnología extranjera por medio de un contrato de licencia (un 66 por 100) 
solamente tenían que pagar royalties. En este caso, el riesgo que asumía el comprador no era muy elevado, dado que únicamente si había producción por parte de la empresa licenciada (la empresa compradora) debían pagarse royalties al licenciador (la empresa vendedora de la tecnología extranjera). Pero si la licenciada no había sido capaz de iniciar la producción, debido, por ejemplo, a dificultades para comprender la tecnología transferida, no tenía que abonar ninguna cantidad. Además, los empresarios españoles habían evaluado previamente las posibilidades de éxito de las tecnologías transferidas, pues se trataba de tecnologías que habían sido aplicadas con anterioridad en los países licenciadores. Por lo tanto, el riesgo implícito en la firma de un contrato de licencia es menor que en la de uno de asistencia técnica, como luego veremos, y que el que implica la inversión en I+D. Como he podido comprobar en la base de datos utilizada, las empresas licenciadas justificaban la firma de un contrato de licencia como una vía para acelerar la llegada de nuevos productos al mercado español y para reducir los riesgos que acarrea la innovación de producto. Además, era una vía de aprendizaje que se transmitía a través de la experiencia de las empresas licenciadoras y una solución para atender la creciente demanda del mercado español, reducir importaciones y ahorrar gastos en I+D. Estos razonamientos son los más mencionados por las empresas instaladas en España para justificar la firma de un contrato de licencia (véase el Cuadro 2) y los que, a mi juicio, más se ajustan a la realidad de aquel momento. Otras razones que pueden encontrarse, y así lo manifestaban los empresarios, eran: diversificar la producción, aumentar la calidad de los productos, incrementar las exportaciones y mejorar la imagen pública de la empresa.

Nuevas variedades de productos fueron introducidas en el mercado español gracias a este tipo de contratos. Se trataba generalmente de bienes en los que los volúmenes de producción eran pequeños y la variable clave para competir no radicaba en la cantidad o el precio, sino en la variedad o la marca (eran productos diferenciados, zapatos por ejemplo). Además, y dada la menor complejidad tecnológica de los bienes producidos mediante un contrato de licencia, el personal extranjero que venía para ayudar a las empresas españolas era habitualmente de un nivel inferior a aquél que un contrato de asistencia técnica implicaba, y permanecía menos tiempo en la empresa española ${ }^{22}$.

Por otra parte, la principal finalidad de los contratos de asistencia técnica era la importación de bienes de producción para la construcción o ampliación de las instalaciones industriales (véase el Cuadro 1). Los bienes de capital necesarios para las nuevas plantas industriales tenían que ser en su mayoría importados, dado su alto

22 La base de datos muestra que el 30,9 por 100 de los 925 contratos de licencia estudiados incluyen visitas del personal español a las fábricas extranjeras, y un 38,1 por 100, visitas del personal extranjero a las plantas españolas para ayudar en la puesta en marcha de las nuevas tecnologías. 


\section{CUADRO 2}

PRINCIPALES RAZONES PARA FIRMAR UN CONTRATO DE TRANSFERENCIA DE TECNOLOGÍA

\begin{tabular}{lcccr}
\hline Razones dadas por las empresas licenciadas & \multicolumn{2}{c}{ Licencias } & \multicolumn{2}{c}{ Asistencia Técnica } \\
& Número & $\%$ del total & Número & $\%$ del total \\
\hline $\begin{array}{l}\text { Competir a nivel internacional } \\
\text { Ahorrar I+D y aprovecharse de la tecnología }\end{array}$ & 38 & 4,11 & 18 & 3,11 \\
extranjera & 107 & 11,57 & 54 & 9,33 \\
Nacionalización y sustitución de importaciones & 140 & 15,14 & 39 & 6,74 \\
$\begin{array}{l}\text { Incapacidad en ausencia de tecnología } \\
\text { extranjera (falta de experiencia) }\end{array}$ & 25 & 2,70 & 114 & 19,69 \\
Hacer frente a la demanda & 166 & 17,95 & 48 & 8,29 \\
Otras razones & 110 & 11,89 & 27 & 4,66 \\
\hline
\end{tabular}

Notas: Algunos contratos dan más de una razón para su firma y no todos ofrecen justificación.

Fuentes: Véase la información suministrada en el texto.

grado de complejidad tecnológica y la imposibilidad de las empresas españolas de fabricarlos. La maquinaria se importaba siempre y cuando no fuera producida en el mercado español. Sin embargo, dichos bienes de capital no constituían la producción final de las empresas que firmaban un contrato de asistencia técnica, estando compuesta aquélla de bienes intermedios y bienes semi-manufacturados (en un 94 por 100 de las ocasiones). Se trataba, en general, de productos homogéneos y maduros; por tanto, la competencia vía precios era muy importante (como ocurre con la innovación de proceso $)^{23}$. Las empresas licenciadas en el caso particular de los contratos de asistencia técnica, como se verá posteriormente, eran predominantemente grandes empresas, por lo que puede afirmarse que en las empresas de mayor tamaño el cambio tecnológico vino más por medio de innovaciones de procesos que por la vía de nuevos productos. La tecnología se transfería gracias a las herramientas y equipos especializados, y al personal extranjero, y la innovación de proceso era la principal tecnología de este tipo de contratos, siendo mucho más importante que la innovación de producto. Se exigía, además, una detallada supervisión y ayuda por parte

23 La etapa de madurez del producto se caracteriza por una cuota de mercado relativamente estable y por una estructura del mercado con un alto grado de concentración, (Klepper, 1996). 
de los suministradores del capital para que la empresa española fuera capaz de adoptar las tecnologías y de utilizar el capital importado. Las principales razones que dan las empresas licenciadas para motivar la firma de un contrato de asistencia técnica son la falta de experiencia y la incapacidad del personal español, así como la necesidad de ahorrar tiempo (véase el Cuadro 2). Debido a la elevada demanda existente en el mercado español, las empresas no tenían tiempo para ocuparse de la propia investigación, pues esto habría requerido, dado el bajísimo nivel y el amplísimo gap respecto de los países más avanzados del cual se partía, muchos años de estudio y experiencia industrial antes de que los nuevos procesos hubieran visto la luz.

Los contratos de asistencia técnica facilitaron a las empresas licenciadas la aplicación de los nuevos procesos de producción, así como la expansión y el abaratamiento de la producción de bienes homogéneos, gracias a la consecución de las economías de escala que las innovaciones de proceso permitían. Las innovaciones de proceso tenían como objetivo principal reducir el coste medio de producción de las empresas, estando las cuantiosas necesidades de capital que los nuevos procesos productivos demandaban fuera del alcance de las empresas más pequeñas. En otras palabras, el coste de adquirir una planta con una escala mínima eficiente constituía una barrera de entrada. El desarrollo de las capacidades de proceso implicaba una gran inversión para pagar el utillaje, el personal y los bienes de equipo extranjeros. Y es que la cantidad a pagar a la empresa licenciadora en el caso de un contrato de asistencia técnica era doble: por una parte, debía pagarse la maquinaria importada y, por otra, la asistencia técnica prestada. Estos pagos, a diferencia de los derivados de un contrato de licencia, tenían que hacerse incluso en el que caso de que no existiera producción alguna por parte de la empresa española, con lo que el riesgo era mayor.

Las grandes empresas necesitaban invertir en nuevos procesos para poder hacer frente a la creciente demanda de productos básicos como soda, ácido sulfúrico, etileno, propileno, amoníaco, etc. Cuanto mayor es la demanda de una empresa mayor es el incentivo para invertir en innovaciones de proceso ${ }^{24}$. Es decir, existe una mayor probabilidad de invertir en innovación de proceso cuando la empresa tiene un mayor número de clientes ${ }^{25}$. Esto se cumple básicamente para productos relativamente homogéneos, donde las economías de escala son significativas así como las inversiones en nuevas plantas y en maquinaria. Se trata, en general, de productos no ensamblados, como por ejemplo, el rayón, el cobre, las fibras sintéticas, el acero, el aluminio, los refinados del petróleo o el cristal. ${ }^{26}$. Algunos ejemplos de empresas españolas que firmaron contratos de asistencia técnica aparecen en el apéndice.

\footnotetext{
$24 \quad$ Klepper (1996), p. 565.

$25 \quad$ Kleppler (1996) y Peters (2000), p. 19.

26 Utterback (1994).
} 
¿Qué relación existe entre estas pautas de adquisición de nuevas tecnologías y el tamaño de las empresas y su poder de monopolio? Veamos lo que la literatura nos dice sobre las conexiones entre la innovación, el tamaño empresarial y la concentración del mercado.

\section{Aspectos teóricos}

Existe una abundante literatura que trata de investigar la relación entre la concentración del mercado y el cambio tecnológico. En su mayoría, se centra en comprobar o refutar la hipótesis debida a Schumpeter: la innovación aumenta más que proporcionalmente con el tamaño de la empresa, y el cambio tecnológico se incrementa con la concentración del mercado ${ }^{27}$. A pesar de los numerosos trabajos al respecto, aún no hay una respuesta unánime acerca del efecto de la concentración sobre la innovación ${ }^{28}$. Así, por ejemplo, algunos estudios han demostrado unos mayores esfuerzos innovadores para las empresas de menor tamaño, pero también para aquéllas de mayor dimensión ${ }^{29}$.

Existen varios argumentos para justificar una relación positiva entre el tamaño de las empresas y la actividad innovadora. Una explicación, que ya fue señalada por Schumpeter, es que las grandes empresas poseen una mayor capacidad para conseguir el cash flow necesario para adoptar proyectos de I+D que suponen un alto riesgo. Otro argumento es que las grandes empresas tienen ventaja en adoptar actividades de $\mathrm{I}+\mathrm{D}$ dado su creciente coste. Estas ventajas de coste se atribuyen tanto a las economías de escala como de diversificación ${ }^{30}$. Además, las empresas con poder de monopolio se apropian mejor de los beneficios de la innovación. El cuarto argumento que se ha señalado es que existen incentivos a la innovación cuando hay posibilidad de conseguir poder de mercado. Las empresas tendrán más incentivos a innovar cuanto mayor sea el poder de mercado anticipado asociado con una innovación ${ }^{31}$. Ello es más difícil de conseguir en un mercado en el que exista una gran competencia, puesto que la competencia podría reducir los incentivos a innovar.

$27 \quad$ Schumpeter (1950).

28 Kamien y Schwartz (1970), Mazzucato (2000), Broadberry y Crafts (2000), Symeonidis (2002). Gayle (2003) ha demostrado la existencia de una relación positiva entre la concentración del mercado y la innovación. Boone (2001) señala que dicha relación depende de cada industria en particular.

29 Pavitt, Robson y Townsend (1987), y Acs y Audretsch (1988).

30 Malecki (1980), Scherer y Ross (1990) y Cohen y Klepper (1996). Sin embargo, Fung (2002) sostiene que, si consideramos las oportunidades tecnológicas que caracterizan a las diferentes industrias, no existe evidencia de la importancia de las economías de escala en la productividad de la actividad investigadora.

31 Peretto (1996), p. 898, ha argumentado que la posesión de poder de mercado puede ser una condición necesaria para la inversión en I+D, pero es la posibilidad de obtener un poder de mercado después de tal inversión la verdadera fuerza determinante de la innovación. Para una mayor información sobre la relación entre poder de mercado e innovación, véase Motta (2004), pp. 58-66. 
Cabe argumentar en contra de lo anteriormente señalado diciendo que la falta de un mercado competitivo o la posesión de poder de mercado desincentiva el cambio tecnológico, dado que la empresa con poder de mercado no se siente amenazada por la entrada de nuevos competidores ${ }^{32}$. Este argumento es válido, como Kumar (1987) ha señalado, cuando las barreras de entrada son altas. Otra conclusión a la que se ha llegado es que las grandes empresas innovan más en las industrias con altos grados de concentración y altas barreras a la entrada, mientras que las pequeñas empresas son más innovadoras en mercados con un alto grado de competencia $^{33}$.

El trabajo empírico más reciente considera la posibilidad de que las relaciones entre la innovación y la estructura de los mercados estén determinadas, más que por el poder de mercado o la estructura de éste, por las características de cada industria en particular: la demanda de mercado del producto (la elasticidad-precio de la demanda), las oportunidades tecnológicas y las condiciones de apropiación ${ }^{34}$. Cohen, Lewin y Mowery, por ejemplo, concluyen que debemos tener en cuenta las diferentes oportunidades tecnológicas que caracterizan a una industria para poder explicar las diferentes propensiones a innovar de las empresas ${ }^{35}$. Al argumento anterior hay que sumar otro, que también suscribe Gifford, según el cual el tamaño empresarial tiene un mayor efecto sobre las altas tasas de innovación en aquellos mercados con bajas oportunidades tecnológicas ${ }^{36}$.

El efecto que la concentración tiene sobre la innovación depende además de la diferenciación del producto ${ }^{37}$. Una importante distinción a este respecto se da entre innovación de proceso e innovación de producto. Como se ha señalado anteriormente, cuanto mayor sea la demanda de producto de una empresa mayor será el incentivo para adoptar innovaciones de proceso $^{38}$. Dicho de otro modo, es más probable que las empresas con un mayor número de clientes inviertan en innovaciones de proceso $^{39}$. Esto es especialmente cierto en el caso de los productos relativamente homogéneos, donde las economías de escala adquieren una gran importancia y donde son precisas grandes inversiones en maquinaria y plantas industriales. Los beneficios que se derivan de la reducción de los costes de producción (innovación

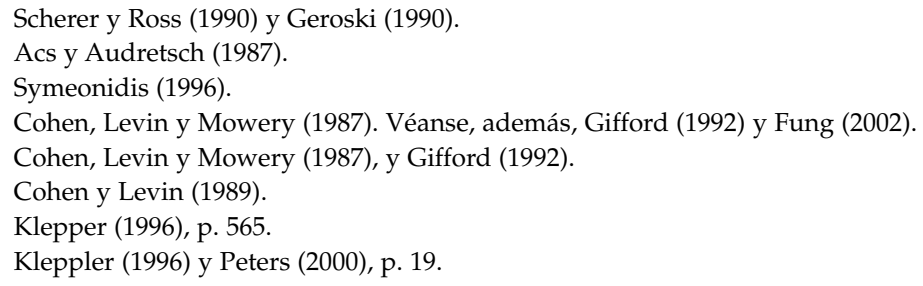


de proceso) son más elevados cuanto mayor es la elasticidad de la demanda ${ }^{40}$. Así, se ha demostrado que cuando los costes de entrada y/o los costes de capital son elevados, la contribución de las pequeñas empresas a la innovación es pequeña y viceversa $^{41}$. Las innovaciones de proceso y la consecución de una planta de un tamaño mínimo eficiente acarrean generalmente elevados costes que constituyen una barrera de entrada para las pequeñas empresas ${ }^{42}$. Existen algunos estudios empíricos que han demostrado que las grandes empresas dedican más recursos de I+D a las innovaciones de proceso que las empresas de menor tamaño ${ }^{43}$.

En resumen, muchas son las razones esgrimidas para intentar explicar la relación entre la concentración empresarial, el poder de mercado y la innovación ${ }^{44}$. El hecho de que haya tantos resultados se debe en parte a cómo se mide la innovación. Por ejemplo, se argumenta que el gasto en $\mathrm{I}+\mathrm{D}$ es una medida muy pobre de la actividad innovadora, puesto que se ha observado que las pequeñas empresas suponen una pequeña parte del total de los insumos (inputs) destinados a la I+D, pero en cambio contribuyen mucho a los outputs de la innovación ${ }^{45}$. Por ejemplo, algunos resultados empíricos concluyen la existencia de una relación directa entre los gastos en I+D y el tamaño de las empresas, pero una relación negativa entre el número de patentes o innovaciones por dólar destinado a I+D y el tamaño de la empresa ${ }^{46}$. Por lo tanto, los diferentes resultados varían según se consideren los inputs destinados a la innovación - por ejemplo, el gasto en $\mathrm{I}+\mathrm{D}$ - o los resultados de las actividades tecnológicas - el número de invenciones que han sido patentadas- como medida de la innovación ${ }^{47}$.

$40 \quad$ Kamien y Schwartz (1970).

41 Rothwell y Dodgson (1996), p. 310.

42 Algunos trabajos - por ejemplo, Lunn (1986) - concluyen que la innovación de proceso conduce a una mayor concentración del mercado, aunque la evidencia no está clara, como demuestra Symeonidis (2002).

$43 \quad$ Scherer (1991), y Pavitt, Robson y Townsend (1987).

44 Los principales trabajos son los de Hall, Griliches y Hausman (1986), Cohen y Levin (1989), y Scherer y Ross (1990).

45 Véanse Acs y Audretsch (1988), Geroski (1990) y Piergiovanni, Santarelli y Vivarelli (1997). La razón dada por los tres últimos autores citados es que las empresas más pequeñas se benefician más que las grandes empresas del conocimiento producido por otras compañías e instituciones. Los spillovers juegan un papel más importante que los gastos en I+D de la industria en la generación de resultados innovadores en el caso de las pequeñas empresas.

46 Pavitt, Robson y Townsend (1987), Acs y Audretsch (1991), y Cohen y Klepper (1996).

$47 \quad$ Así lo indica Vossen (1999). El autor encuentra una relación positiva entre la concentración industrial y los gastos en I+D (que es una medida de los inputs que se dedican a la innovación) y una relación negativa si tenemos en cuenta el número de innovaciones (una medida del output de la innovación). 


\section{Variables e hipótesis}

En el presente epígrafe se pretende comprobar si existe una relación entre el tamaño de las empresas, el poder de monopolio y la inversión en la adopción de nuevas tecnologías. El poder de monopolio puede ser debido a la existencia de barreras legales a la entrada de nuevos competidores. El objetivo es estudiar cómo las barreras legales a la entrada que fueron establecidas por la regulación industrial española en la época franquista afectaron a la política de adopción de la tecnología extranjera. Las principales variables explicativas, como puede verse en el Cuadro 3, son: el tamaño de las empresas (TAMAÑO), el grado de regulación a la entrada de nuevas empresas (REGUL) y el tipo de producto (HOMOG) ${ }^{48}$. La variable REGUL se ha dividido en tres variables dummies $^{49}$. Las variables de control introducidas en el modelo son: DESP67 (indicativa del incremento del grado de regulación industrial en el mercado español después del decreto 1.776 de julio de 1967); LOGPIB (una variable indicativa del nivel de la demanda española) y cinco variables sectoriales: minería y construcción, naval, química, textiles, alimentación y otros, e industria siderometalúrgica.

Se espera encontrar las siguientes relaciones ${ }^{50}$ :

Hipótesis 1. Las empresas de mayor tamaño (TAMAÑO) tienen mayor probabilidad de invertir más en nuevas tecnologías, dada su mayor capacidad para invertir en proyectos que requieren una elevada cantidad de dinero.

La relación entre el nivel de competencia y la innovación no está clara. La variable REGUL podría ser una proxy para medir el grado de competencia, dado que, como han demostrado Buesa y Pires, los sectores más regulados por el Estado eran aquéllos con un mayor grado de concentración (Cuadro 4).

La probabilidad de encontrar mayores gastos en nuevas tecnologías podría aumentar en los sectores con un menor grado de competencia, ya que cuanto mayor es el poder de mercado mayor será el incentivo a invertir en nuevas tecnologías. Pero también es cierto que la ausencia de competencia desincentiva la innovación. Por lo tanto, a priori no esperamos ningún resultado concluyente.

48 Podríamos esperar una relación positiva entre las variables HOMOG y REGUL. Sin embargo, la evidencia empírica sobre la relación entre la competencia vía precios y una mayor concentración no es definitiva. Véase Symeonidis (2002), p. 88.

49 Las variables dummies son REGUL1, REGUL2 y REGUL3. La variable REGUL3 toma el valor 1 para aquellas industrias que requerían autorización previa para el establecimiento o ampliación de las instalaciones.

50 Hace dos décadas, Braña, Buesa y Molero (1984) señalaron, a partir de una base de datos compuesta por 1.373 contratos firmados entre 1974 y 1976, que los pagos por la transferencia de tecnología (pagos por licencias y por asistencia técnica) eran principalmente efectuados por las mayores empresas y por aquéllas con una mayor participación de capital público y de capital extranjero. Los autores no aplicaron ningún modelo econométrico, describiendo simplemente la base de datos. La relación de dichos contratos, no así su contenido, apareció publicada en la revista Economía Industrial. 


\section{CUADRO 3}

LISTA DE VARIABLES EXPLICATIVAS

\section{Tamaño de empresa e innovación:}

TAMAÑO: Log del capital social de las empresas.

\section{Concentración del mercado (barreras a la entrada) e innovación:}

REGUL1 (Regulación Industrial 1): Variable dummy igual a 1 para aquellas industrias que estaban liberalizadas desde el punto de vista de la regulación industrial. REGUL2 (Regulación Industrial 2): Variable dummy que toma el valor 1 para aquellas industrias a las que se les exigía un mínimo de condiciones técnicas y de tamaño para el establecimiento y ampliación de industrias. DESP67: Variable dummy igual a 1 si el contrato fue firmado después del año 1967.

\section{Variables a nivel industrial:}

HOMOG: Variable dummy igual a 1 si el bien producido con la tecnología extranjera es un bien homogéneo. MINERÍA\&CONSTR: Variable dummy igual 1 si la tecnología que se transfiere hace referencia al sector de la construcción o de la minería. NAVAL: Variable dummy que toma el valor 1 si la tecnología que se transfiere hace referencia al sector naval. QUíMICA: Variable dummy que toma el valor 1 si la tecnología que se transfiere hace referencia al sector químico. TA: Variable dummy que toma el valor 1 si la tecnología que se transfiere hace referencia al sector textil, alimentación y otros.

\section{Ciclo económico e innovación:}

LOGPIB: Log del PIB español a precios de mercado (precios constantes).

Nota: La información referente a la regulación industrial ha sido tomada del trabajo de Buesa y Pires (2002). Dichos autores han clasificado las diferentes actividades industriales según los tres grupos de regulación industrial (liberalizadas, aquéllas con un mínimo de condiciones técnicas y de tamaño, y aquéllas que requerían autorización previa). Los datos del PIB son los de Prados de la Escosura (1995). La información sobre el resto de las variables procede de la base de datos recopilada a partir de los contratos de transferencia de tecnología que fueron firmados entre las empresas españolas y las empresas extranjeras.

Tampoco está clara, por la misma razón que en el caso anterior, la probabilidad de encontrar empresas con mayores inversiones en nuevas tecnologías después del año 1966, cuando por el Decreto 1.776 de julio de 1967 se incrementó el nivel de regulación industrial. 


\section{CUADRO 4}

RELACIÓN ENTRE TECNOLOGÍA, TASAS DE CRECIMIENTO Y GRADO DE OLIGOPOLIO EN SECTORES CON ALTA REGULACIÓN INDUSTRIAL. ESPAÑA, 1959-1972

\begin{tabular}{lccccccc}
\hline \multicolumn{1}{c}{ Sectores } & \multicolumn{2}{c}{ Complejidad tecnológica } & $\begin{array}{c}\text { Tasas de } \\
\text { crecimiento } \\
\text { sectoriales }\end{array}$ & $\begin{array}{c}\text { Oligo- } \\
\text { polio }\end{array}$ \\
\hline & Muy alta & Alta & Media & Baja & Alta & Baja & \\
\hline Minerales metálicos & - & - & $X$ & - & $X$ & - & $X$ \\
Papel & - & - & - & $X$ & $X$ & - & $X$ \\
Textiles & - & - & - & $X$ & - & $X$ & - \\
Calzado & - & - & - & $X$ & $X$ & - & - \\
Industria siderometalúrgica & - & - & $X$ & - & $X$ & - & $X$ \\
Metales no férreos & - & - & $X$ & - & $X$ & - & $X$ \\
Productos químicos & & & & & & & \\
para consumo final & - & $X$ & - & - & $X$ & - & - \\
Minería & - & $X$ & - & - & - & - & $X$ \\
Naval & - & - & $X$ & - & $X$ & - & $X$ \\
Automóviles & - & $X$ & - & - & $X$ & - & $X$ \\
Energía eléctrica & - & $X$ & - & - & $X$ & - & - \\
Material electrónico & $X$ & - & - & - & $X$ & - & $X$ \\
Maquinaria eléctrica & $X$ & - & - & - & $X$ & - & - \\
Refinado de petróleo & $\mathrm{X}$ & - & - & - & $X$ & - & $X$ \\
\hline
\end{tabular}

Notas y Fuentes: Regulación industrial alta: aquellas industrias a las que se les exigía autorización previa para el establecimiento, traslado o ampliación de las plantas industriales. Las tasas de crecimiento se refieren al período 1958-1972, véase Buesa y Pires (2002). Véase Molero (1982) para la complejidad tecnológica. La información referente a los sectores oligopolísticos ha sido tomada de Buesa y Molero (1988), p. 84. Los autores consideran el grado de concentración, el tamaño de las empresas y el número de competidores para saber cuáles son los sectores con una estructura oligopolística.

Hipótesis 2. La probabilidad de realizar mayores gastos en las nuevas tecnologías aumenta si la producción final de la empresa compradora de tecnología extranjera es un bien homogéneo (HOMOG). Cuando se trata de un producto maduro, las empresas invierten en innovaciones de proceso, que requieren cuantiosas necesidades de capital, con el objetivo de incrementar la escala de producción y de conseguir economías de escala ${ }^{51}$. 
Hipótesis 3. Cuanto mayor es la demanda de productos homogéneos mayor será la probabilidad de que aumenten los gastos en la adopción de nuevas tecnologías, puesto que mayores serán los incentivos para invertir en éstas ${ }^{52}$. Se ha utilizado la variable proxy LOGPIB a precios de mercado para medir el nivel de la demanda de las empresas compradoras de tecnología extranjera. Se espera además que cuanto mayor sea la tasa de crecimiento mayor será el gasto en nuevas tecnologías ${ }^{53}$.

\section{Especificación del modelo y resultados empíricos}

La variable dependiente del análisis, que puede ser interpretada como el gasto de las empresas españolas en nuevas tecnologías, toma los siguientes valores:

1: si se trata de un contrato de asistencia técnica extranjera (mayores gastos en nuevas tecnologías).

0: $\quad$ si estamos ante un contrato de licencia de tecnología extranjera (menores gastos en la adquisición de nuevas tecnologías).

El modelo de regresión es el siguiente:

$E=b_{0}+b_{1}$ TAMA $O+b_{2} R E G U L 1+b_{3} R E G U L 2+b_{4} H O M O G+b_{5} L O G P I B+\sum_{6}^{9} b_{j} S E C T O R+b_{10} D E S P 67+e_{i}$

Todas las variables tienen el signo correcto y son estadísticamente significativas, en su mayoría al nivel del 1 por 100 (véase el Cuadro 5). El coeficiente de la variable TAMAÑO es positivo y muy significativo. Como se esperaba, los gastos en tecnología extranjera son relativamente más importantes para las empresas de mayor tamaño. El hecho de que la variable REGUL1 sea estadísticamente significativa y de signo negativo indica que las empresas de los sectores liberalizados tienen una menor propensión a invertir en nuevas tecnologías que las empresas de los sectores regula$\operatorname{dos}^{54}$. El coeficiente de la variable HOMOG indica que la probabilidad de invertir en nuevas tecnologías depende también del tipo de bien producido. Cuando se trata de la fabricación de bienes homogéneos los gastos en la inversión de nuevas tecnologías también se incrementan en relación con aquellas empresas productoras de bienes de capital y de bienes de consumo. El signo de la variable LOGPIB es positivo, lo que indica que el crecimiento de la demanda influye positivamente en los gastos de adquisición de nuevas tecnologías.

Cohen, Lewin y Mowery (1987).

Schmookler (1966).

Hay algunos estudios que defienden una relación positiva entre el nivel de regulación y la adopción de nuevas innovaciones. Véase Baker (2001). 
CUADRO 5

REGRESIONES LOGÍSTICAS:

CONTRATO DE ASISTENCIA TÉCNICA (=1) VERSUS CONTRATO DE LICENCIA (=0)

\begin{tabular}{|c|c|c|c|c|}
\hline \multirow{2}{*}{$\begin{array}{l}\text { Variables explicativas } \\
\text { Constante }\end{array}$} & \multicolumn{2}{|c|}{ Modelo 1} & \multicolumn{2}{|c|}{ Modelo 2} \\
\hline & $-50,154^{\star \star *}$ & $(17,100)$ & 23,354 & $(26,460)$ \\
\hline TAMAÑO & 0,354 *** & $(0,037)$ & $0,350^{* \star *}$ & $(0,038)$ \\
\hline REGUL1 & $-0,875^{\star \star}$ & $(0,382)$ & $-0,876^{\star \star}$ & $(0,400)$ \\
\hline REGUL2 & $-1,021^{\star}$ & $(0,438)$ & $-0,917^{*}$ & $(0,496)$ \\
\hline HOMOG & 1,150 *** & $(0,216)$ & $1,332^{\star \star *}$ & $(0,250)$ \\
\hline LOGPIB & 5,911 ** & $(2,383)$ & $-4,375$ & $(3,700)$ \\
\hline MINERÍA\&CONSTR & & & $2,554^{\star \star \star}$ & $(0,585)$ \\
\hline NAVAL & & & $1,029^{*}$ & $(0,577)$ \\
\hline QUÍMICA & & & $-0,050$ & $(0,277)$ \\
\hline TA & & & $-0,073$ & $(0,363)$ \\
\hline DESP67 & 0,844 ** & $(0,356)$ & $-111,206^{\star \star *}$ & $(34,012)$ \\
\hline LOGCAP*DESP67 & & & $15,559^{* \star *}$ & $(4,718)$ \\
\hline Número de observaciones & 776 & & 776 & \\
\hline-2 Log de la verosimilitud & 563,393 & & 531,374 & \\
\hline $\mathrm{Chi}^{2}$ & 473,929 & & 505,948 & \\
\hline Prob>Chi ${ }^{2}$ & 0,000 & & 0,000 & \\
\hline \multicolumn{5}{|c|}{ 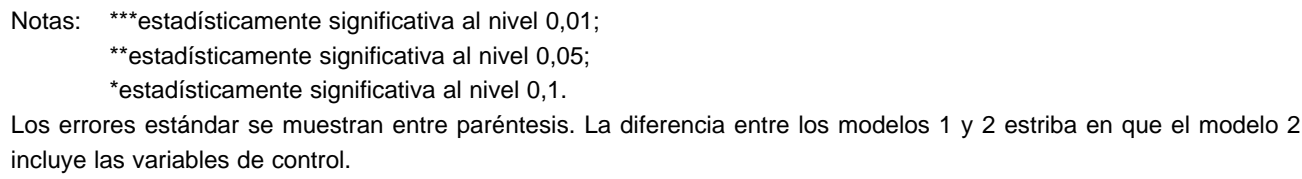 } \\
\hline
\end{tabular}

El Cuadro 6 muestra la probabilidad de la firma de un contrato de asistencia técnica (más gastos en la adopción de nuevas tecnologías) frente a un contrato de licencia (menor inversión). Se muestra una comparación de las ratios entre las probabilidades antes y después de un cambio incremental en la variable independiente. Como puede observarse, el cociente de probabilidades de que una empresa gaste más en nuevas tecnologías es 1,42 veces mayor cuando la empresa es de mayor tamaño. En cambio, en los sectores liberalizados el cociente de probabilidades es inferior respecto al de los sectores que se encuentran bajo el sistema administrativo de autorización previa (modelo 1). Si se trata de un producto homogéneo, entonces un mayor gasto en cambio tecnológico es 3,15 veces más probable que si se tratase de un producto 


\section{CUADRO 6}

REGRESIONES LOGÍSTICAS:

CONTRATO DE ASISTENCIA TÉCNICA VERSUS CONTRATO DE LICENCIA (ODDS RATIO)

\begin{tabular}{|c|c|c|c|c|}
\hline \multirow{2}{*}{$\begin{array}{l}\text { Variables explicativas } \\
\text { TAMAÑO }\end{array}$} & \multicolumn{2}{|c|}{ Modelo 1} & \multicolumn{2}{|c|}{ Modelo 2} \\
\hline & $1,425^{\star \star \star}$ & $(0,0526)$ & $1,418^{\star \star *}$ & $(0,053)$ \\
\hline REGUL1 & $0,417^{\star *}$ & $(0,1590)$ & $0,416^{\star *}$ & $(0,1666)$ \\
\hline REGUL2 & $0,360^{\star *}$ & $(0,1578)$ & $0,399^{*}$ & $(0,1982)$ \\
\hline HOMOG & $3,156^{\star \star \star}$ & $(0,682)$ & $3,788^{\star \star \star}$ & $(0,948)$ \\
\hline LOGPIB & $369,048^{\star \star}$ & $(879,548)$ & 0,0125 & $(0,0465)$ \\
\hline MINERÍA\&CONSTR & & & $12,857^{\star \star \star}$ & $(7,522)$ \\
\hline NAVAL & & & $2,799^{\star}$ & $(1,615)$ \\
\hline QUÍMICO & & & 0,951 & $(0,263)$ \\
\hline TA & & & 0,929 & $(0,337)$ \\
\hline DESP1967 & $2,326756^{\star *}$ & $(0,8273538)$ & $5,04 \mathrm{e}-49 * \star \star$ & $(1,72 \mathrm{e}-47)$ \\
\hline LOGPIB`DESP67 & & & $5719453^{\star \star \star}$ & $(2,70 \mathrm{e}+07)$ \\
\hline Número de observaciones & 776 & & 776 & \\
\hline-2 Log de la verosimilitud & 563,393 & & 531,734 & \\
\hline Pseudo $\mathbf{R}^{2}$ & 0,4569 & & 0,4877 & \\
\hline $\mathrm{Chi}^{2}$ & 473,929 & & 505,948 & \\
\hline Prob $>\mathrm{Chi}^{2}$ & 0,000 & & 0,000 & \\
\hline
\end{tabular}

Notas: $\quad * * *$ estadísticamente significativa al nivel 0,01;

**estadísticamente significativa al nivel 0,05;

*estadísticamente significativa al nivel 0,1.

Los errores estándar se muestran entre paréntesis. El odds asociado a un suceso es el cociente entre la probabilidad de que ocurra frente a la probabilidad de que no ocurra: odds=p/1-p, siendo $\mathrm{p}$ la probabilidad del suceso. El odds ratio (o razón de probabilidades) de alguna manera cuantifica cuánto más probable es la aparición de la firma de un contrato de asistencia técnica con relación a contrato de licencia cuando la empresa pertenece a un sector regulado, o es una empresa grande, etc.

diferenciado. Se observa también que una mayor inversión en nuevas tecnologías es menos probable (coeficiente menor que 1) en los sectores liberalizados, en los sectores no muy regularizados, y en los sectores químico, textil, alimentación y otros ${ }^{55}$.

55 Se han obtenido también regresiones logísticas para cada industria. Los resultados obtenidos indican que las variables TAMAÑO y DESP1967 son estadísticamente significativas en el caso del sector textil, alimentación y diversas. Las variables TAMAÑO y HOMOG son estadísticamente significativas en el caso de la industria química, y la variable HOMOG lo es para la construcción y minería. 


\section{Conclusión}

En estas páginas se ha intentado arrojar nueva luz sobre la relación entre la estructura del mercado, el tamaño de las empresas y el gasto en nuevas tecnologías, a partir de una muestra de datos para el caso español en el período 1959-1973. El debate sobre la existencia de una relación positiva o negativa entre dichas variables aún no está resuelto. Los resultados obtenidos en este trabajo están en concordancia con la hipótesis que en su día anunció Schumpeter: las industrias más concentradas tienen una mayor probabilidad de invertir en nuevas tecnologías. Los resultados aquí obtenidos son válidos para un país relativamente atrasado, con una economía muy protegida frente al exterior, con un alto grado de regulación estatal y con bajos niveles de competencia en el mercado interior.

Se han comprobado tres hipótesis. La primera establece una relación positiva entre la probabilidad de mayores gastos en la compra de nuevas tecnologías y el tamaño de la empresa. Segundo, se ha estudiado qué relación existe entre la concentración del mercado y los gastos en la incorporación del cambio tecnológico. Los resultados indican que dicha relación es positiva, es decir, las industrias más reguladas son aquéllas en las que el gasto en nuevas tecnologías fue más elevado. Por último, la tercera hipótesis que se ha comprobado es la existencia de mayores gastos en innovación para aquellas empresas que producen bienes homogéneos. Este resultado confirma la hipótesis de Cohen y Klepper de la existencia de una relación positiva entre los beneficios que se derivan de la innovación y el tamaño empresarial y que dicha relación es mayor para las innovaciones de proceso que para las innovaciones de producto ${ }^{56}$.

Los resultados obtenidos indican una mayor relación entre el tamaño empresarial y la inversión en nuevas tecnologías que entre esta variable y la concentración del mercado. El trabajo empírico también concluye que hay otras variables, como el grado de diferenciación de producto, que pueden afectar a la actividad innovadora. Las empresas que producen bienes homogéneos tienen mayor probabilidad de invertir mayores cantidades en la compra de nuevas tecnologías que las empresas que producen bienes con un alto grado de diferenciación de producto. Otra de las conclusiones obtenidas es que el gasto en innovación varía según el tipo de sector. Así, en el sector naval, de la construcción y de la minería, sectores muy protegidos y regulados por el Estado español, se ha encontrado una mayor inversión en la adquisición de nuevas tecnologías.

Por lo tanto, se puede concluir afirmando que la elección entre un contrato de asistencia técnica (con mayores gastos en nuevas tecnologías) o un contrato de licencia (sinónimo de menores gastos en tecnología), en el caso de la economía española en el período 1959-1973, dependió de la estructura del mercado, la política industrial y las características de cada industria. Las industrias con un mayor grado de concentración 
(que eran las más reguladas por el gobierno) gastaron mucho más en nuevas tecnologías a través de los contratos de asistencia técnica. Su objetivo era la producción de bienes intermedios y semi-manufacturados. Además, la política industrial favoreció a los grupos con un mayor poder de mercado y los incentivó para que adoptaran esta política de innovación mediante la transferencia de tecnologías extranjeras. El marco institucional no estimuló a las grandes empresas para que invirtieran en innovaciones propias, ya que la imitación permitía mantener el status quo de los grupos económicos de poder. Por consiguiente, el cambio tecnológico a través de la compra de tecnología extranjera parece haber influido en la supervivencia de los oligopolios y de los grupos económicos de poder. Las empresas que más gastaron en la compra de tecnología extranjera fueron a su vez las que estaban más reguladas y las que recibían más ayudas estatales. La importación de maquinaria de alto grado de complejidad tecnológica, necesaria para la producción de bienes intermedios de las empresas más favorecidas por el Estado, fue facilitada por el gobierno español con unos bajos aranceles. En cambio, la producción de bienes intermedios estaba muy protegida frente a la competencia exterior.

En definitiva, España ha sido un país imitador porque la ausencia de un verdadero marco competitivo no favoreció la inversión en innovación nacional en un mercado con altos grados de protección y de intervención estatal. No se puede culpar a las empresas extranjeras del hecho de que España no haya sido un país innovador. La compra de tecnología extranjera era la opción más rentable, más rápida y más segura, dado que el riesgo asociado a la adopción de tecnologías extranjeras siempre es menor al de la inversión en innovaciones domésticas. Debido a que las empresas con un mayor grado de concentración y de mayor tamaño (precisamente las que mayor probabilidad tienen de invertir en I+D) estuvieron protegidas por el Estado, el gasto español en tecnologías propias fue de muy escasa cuantía ya que no existían incentivos para ello, pues imitando estas grandes empresas podían seguir manteniendo su poder de mercado. El hecho de que España haya sido siempre un país seguidor es el precio que ha habido que pagar por haber adoptado la imitación como una estrategia de crecimiento económico.

\section{Bibliografía}

ACS, Zoltan y AUDRETSCH, David (1987): “Innovation, Market Structure and Firm Size", Review of Economics and Statistics, 69, 4, pp. 567-574.

-(1988): "Innovation in large and small firms: An Empirical Analysis", American Economic Review, 78, 4, pp. 678-690.

-(1991): "R\&D, firm size, and innovative activity", en ACS, Zoltan y AUDRETSCH, David (eds.), Innovation and Technological Change: An international Comparison, New York, Harvester Wheatsheaf. 
ALONSO, José Antonio (1993 [1988]): "El Sector Exterior", en García Delgado, José Luis (dir.), España. Economía, Madrid, Espasa Calpe, pp. 383-478.

ARCHIBUGI, Danielle; CESARATTO, Sergio y SIRILLI, Giorgio (1991): “Sources of Innovative Activities and Industrial Organization in Italy", Research Policy, 20, pp. 299-313.

BAKER, Laurence (2001): "Manager Care and Technology Adoption in Health Care: Evidence from Magnetic Resonance Imaging", Journal of Health Economics, 20, 3, pp. 395-421.

BARCIELA, Carlos (2002): “Guerra civil y primer franquismo”, en COMÍN, Francisco, HERNÁNDEZ, Mauro, y LLOPIS, Enrique (eds.), Historia económica de España. Siglos X-XX, Barcelona, Crítica, pp. 331-367.

BARCIELA, Carlos, LÓPEZ, Ma Inmaculada, MELGAREJO, Joaquín y MIRANDA, José Antonio (2001): La España de Franco (1939-1975), Madrid, Síntesis.

BOONE, Jan (2001): "Intensity of competition and the incentive to innovate", International Journal of Industrial Organization, 19, pp. 705-726.

BRAGA, Helson y WILLMORE, Larry (1991): “Technological imports and technological effort: An analysis of their determinants in Brazilian firms", Journal of Industrial Economics, 39, 4, June, pp. 421-433.

BRAÑA, Francisco J., BUESA, Mikel, y MOLERO, José (1984): El Estado y el cambio tecnológico en la industrialización tardía: un análisis del caso español, México, FCE.

BROADBERRY, Stephen, y CRAFTS, Nick (2000): “Competition and innovation in 1950's Britain", Working Paper in Economic History, 57, London School of Economics.

BUESA, Mikel (1982): El Estado en el proceso de industrialización: contribución al estudio de la política industrial española en el período 1939-1963, Tesis Doctoral, Facultad de Ciencias Económicas y Empresariales, Madrid, Universidad Complutense.

BUESA, Mikel, y PIRES Luis Eduardo (2002): "Intervencionismo estatal durante el franquismo tardío: la regulación de la inversión industrial en España (1963-1980)", Revista de Historia Industrial, 21, pp. 159-198.

CALVO, José L. (2000): ¿Son las Actividades de I+D una buena aproximación a la Innovación Tecnológica?, Madrid, UNED.

CATALÁN, Jordi (1995): La economía española y la Segunda Guerra Mundial, Barcelona, Ariel.

CEBRIÁN, Mar (2004): “Technological Imitation and Economic Growth during the Golden Age, 1959-1973", Tesis Doctoral, Instituto Universitario Europeo.

COHEN, Wesley, y KLEPPER, Steven (1996): “A Reprise of Size and R\&D”, The Economic Journal, 106, 437, pp. 925-951.

COHEN, Wesley, y LEVIN, Richard C. (1989): “Empirical Studies of Innovation and Market Structure", en SCHAMLENSEE, Richard, y WILLIG, Robert (eds.), Handbook of Industrial Organization, vol. 2, Amsterdam, North Holland, pp. 1.059-1.106.

COHEN, Wesley, LEVIN, Richard C., y MOWERY, David C. (1987): "Firm Size and R\&D intensity: A Re-examination", Journal of Industrial Economics”, 35, pp. 543-565. 
COMÍN, Francisco (1997): “El Estado, la rigidez de los mercados y la convergencia en el siglo XX", ponencia presentada al VI Congreso de la Asociación Española de Historia Económica, Gerona, 15-17 septiembre.

DOSI, Giovanni (1988): "Sources, Procedures, and Microeconomic Effects on Innovation", Journal of Economic Literature, 36, pp. 1120-71.

FRAILE, Pedro (1991): Industrialización y grupos de presión. La economía política de la protección en España 1900-1950, Alianza Editorial, Madrid.

-(1999): "Spain: Industrial Policy Under Authoritarian Policies", en FOREMANPECK, James, y FEDERICO, Giovanni, European Industrial Policy. The TwentiethCentury Experience, New Cork, Oxford University Press.

FUNG, Mark K. (2002): "Technological opportunity and economies of scale in Research Productivity: A Study of Three Global Industries", Review of Industrial Organization", 21, 4, pp. 419-36.

COMÍN, Francisco, y GÁLVEZ, Lina (2003): “Multinacionales, atraso tecnológico y marco institucional. Las nacionalizaciones de empresas extranjeras durante la autarquía franquista", Cuadernos de Economía y Dirección de la Empresa, 17, pp. 139179.

GAYLE, Philip G. (2003): "Market Concentration and Innovation: New Empirical Evidence on the Schumpeterian Hypothesis", Working Paper, Kansas State University, Manhattan.

GEROSKI, Paul (1990): "Innovation, Technology Opportunity and Market Structure”, Oxford economic papers, 42, 3, pp. 586-602.

-(1991): "Entry and the rate of innovation", Economic Innovation and New Technology, 1, pp. 203-214.

GIFFORD, Sharon (1992): "Innovation, Firm Size and Growth in a Centralized Organization", Rand Journal of Economics, 23, 2, pp. 284-98.

GONZÁLEZ, Manuel Jesús (1979): La economía política del franquismo (1940-1970). Dirigismo, mercado y planificación, Madrid, Tecnos.

HALL, Bronwyn, y KHAN, Beethika (2003): Adoption of New Technology, en JONES, Derek (ed.), New Economy Handbook, Cambridge, MA, Elsevier.

HALL, Bronwyn, GRILICHES, Zvi, y HAUSMAN, Jerry A. (1986): "Patents and R\&D: Is there a Lag?", International Economic Review, 27, pp. 265-83.

KAMIEN, Morton, y SCHWARTZ, Nancy (1970): "Market Structure, Elasticity of Demand, and Incentive to Invent", Journal of Law and Economics, 90, pp. 245-260.

KLEPPER, Steven (1996): "Entry, Exit, Growth and Innovation over the Product Life Cycle", American Economic Review, 86, 3, pp. 562-583.

KUMAR, Nagesh (1987): “Technology Imports and Local Research and Development in Indian Manufacturing", The Developing Economies, 25, pp. 220-233.

LÓPEZ, Santiago (1992): "Un sistema tecnológico que progresa sin innovar. Aproximación a las claves de la Tercera Revolución Tecnológica en España", Ekonomiaz, 22, pp. 30-55. 
LÓPEZ, Santiago, y VALDALISO, Jesús María (2001): “Cambio tecnológico y crecimiento económico en España en la segunda mitad del siglo XX: indicadores y polémicas", Revista de Historia Industrial, 19-20, pp. 319-39.

LUNN, John (1986): "An Empirical Analysis of Process and Product Patenting: a simultaneous equation framework", Journal of Industrial Economics, 34, pp. 319-328.

MALECKI, Edward J. (1980): “Firm Size, Location, and Industrial R\&D: a Disaggregated Analysis", Review of Business and Economic Research, 16, pp. 29-42.

MALUQUER DE MOTES, Jordi (2000): “Organización industrial y crecimiento económico en la España contemporánea: tecnología y estructura productiva", presentado al Congreso Internacional Historia de la Empresa Ibérica, Fundación Rei Alfonso Henriques, Zamora, 18-19 octubre.

MARTÍN ACEÑA, Pablo, y COMÍN, Francisco (1991): INI, 50 años de industrialización en España, Madrid, Espasa Calpe.

MARTÍN GONZÁLEZ, Carmela, y RODRÍGUEZ ROMERO, Luis (1979): “Análisis comparado de la intervención del sector público en España en los procesos de generación y difusión de tecnología", Información Comercial Española, 552, pp. 19-33.

MAZZUCATO, Mariana (2000): Firm Size, Innovation and Market Structure. The Evolution of Industry Concentration and Instability, London, Edward Elgar.

MOLERO, José (1982): Tecnología e Industrialización, Madrid, Pirámide.

MOLERO, José, y MIKEL, Buesa (1995): "Innovación y Cambio Tecnológico", en GARCÍA DELGADO, José Luis (dir.), Lecciones de Economía Española, Madrid, Civitas.

MOTTA, Massimo (2004): Competition Policy. Theory and Practice, Cambridge MA, Cambridge University Press.

NAPOLITANO, Giovanni (1991): “Industrial research and sources of innovation: a crossindustry analysis of Italian manufacturing firms", Research Policy, 20, pp 171-178.

PAVITT, Keith, ROBSON, Michael, y TOWNSEND, Joe (1987): “The Size Distribution of Innovating Firms in the UK: 1945-1983", Journal of Industrial Economics, 35, pp. 297316.

PERETTO, Pietro F. (1996): "Sunk costs, Market Structure, and Growth", International Economic Review, 37, 4, pp. 895-923.

PETERS, Juergen (2000): “Buyer Market Power and Innovative Activities. Evidence for the German Automobile Industry", Review of Industrial Organization, 16, pp. 13-38.

PIERGIOVANNI, Roberta; SANTARELLI, Enrico y VIVARELLI, Marco (1997): “From Which Source Do Small Firms Derive Their Innovative Inputs? Some Evidence from Italian Industry", Review of Industrial Organization, 12, pp. 243-258.

PIRES, Luis Eduardo (2003): Regulación industrial y atraso económico en la dictadura de Franco, Madrid, Dykinson, Servicio de Publicaciones de la Universidad Rey Juan Carlos.

ROLDÁN, Santiago, MUÑOZ, Juan, y SERRANO, Ángel (1978): La internacionalización del capital en España 1959-75, Madrid, Edicusa.

ROTHWELL, Roy, y DODGSON, Mark (1996): "Innovation and Size of Firm", en 
DODGSON, Mark, y ROTHWELL, R. (eds.), The Handbook of Industrial Innovation, Cheltenham, Edward Elgar.

SÁIZ, Juan Patricio (1999): Invención, patentes e innovación en la España contemporánea, Madrid, OEPM-MIE.

SANZ, Luis, y LÓPEZ, Santiago (1997): “Política Tecnológica Versus Política Científica durante el Franquismo", Quaderns d'Historia de l'Enginyieria, II, pp. 77-118.

SCHERER, Frederick M. (1991): "Changing perspectives on the firm size problem", en ACS, Zoltan J., y AUDRETSCH, David B. (eds.), Innovation and technological Change: An International comparison, New York, Harvester Wheatsheaf.

SCHERER, Frederick M., y ROSS, David (1990): Industrial Market Structure and Economic Performance, Boston, Houghton Mifflin Company.

SCHMOOKLER, Jacob (1966): Invention and Economic Growth, Cambridge, Harvard University Press.

SCHUMPETER, Joseph A. (1950 [1942]): Capitalism, Socialism, and Democracy, New York, Harper \& Row.

SERRANO SANZ, José María (1997): "Sector exterior y desarrollo de la economía española contemporánea", Papeles de Economía Española, 73, pp. 308-335.

SERRANO SANZ, José María, y PARDOS, Eva (2002): “Los años de crecimiento del franquismo (1959-1975)" en COMÍN, Francisco, HERNÁNDEZ, Mauro, y LLOPIS, Enrique (eds.), Historia Económica de España, Siglos X-XX, Barcelona, Crítica, pp. 369-395.

SIDDHARTHAN, Natteri S. (1992): “Transaction Costs, Technology Transfer, and Inhouse R\&D: A Study of the Indian Private Corporate Sector", Journal of Economic Behaviour and Organization, 18, pp. 265-271.

SIDDHARTHAN, Natteri S., y AGARWAL, Rajshree (1992): "Determinants of R\&D Decisions: A Cross Section Study of Indian Private Corporate Firms", Economics of Innovation and New Technology, 2, pp. 103-110.

STEINKAMP, Gunther, y GARCÍA VIÑUELA, Enrique (1977): “Plan de Estabilización y Política de Importaciones (1959-1967)”, Investigaciones Económicas, 3, mayo-agosto, pp. 27-65.

SYMEONIDIS, George (1996): "Innovation, firm size and market structure: Schumpeterian hypotheses and some new themes", OECD Economic Studies, 27, pp. 35-70.

-(2002): The Effects of Competition. Cartel Policy and the Evolution of Strategy and Structure in British Industry, Cambridge, The MIT University Press.

TIROLE, Jean (1988): The Theory of Industrial Organization, Cambridge, The MIT University Press.

UTTERBACK, James (1994): Mastering the Dynamics of innovation: How Companies Can Seize Opportunities in the Face of Technological Change, Cambridge, MA, Harvard Business School Press.

VALDALISO, Jesús María (2004): “Grupos empresariales y relaciones Banca-Industria en España durante el franquismo: una aproximación macroeconómica", Información Comercial Española, 812, pp. 163-178. 
VIÑAS Ángel, VIÑUELA, Julio, EGUIDAZU, Fernando, FERNÁNDEZ PULGAR, Carlos, y FLORENSA, Senén (1979): Politica comercial exterior de España (1931-1975), Madrid, Banco Exterior de España, Servicio de Estudios Económicos, Vol. II.

VISCUSI, Kip, VERNON, John, y HARRINGTON, Joseph E. (1995 [1992]): Economics of Regulation and Antitrust, Cambridge MA, The MIT Press [2 ${ }^{\mathrm{a}}$ edición].

VOSSEN, Robert (1999): "Market Power, Industrial Concentration and Innovative Activity", Review of Industrial Organisation, 15, pp. 367-378.

\section{APÉNDICE}

ALGUNOS EJEMPLOS DE EMPRESAS ESPAÑOLAS QUE FIRMARON UN CONTRATO DE ASISTENCIA TÉCNICA

\begin{tabular}{lccc}
\hline Nombre de & Número & Nombre de & Número \\
la empresa & de & la empresa & de \\
licenciada & contratos & licenciada & contratos \\
\hline
\end{tabular}

Abonos Complejos del Sureste (ASUR, S.A.) 1 Abonos de Unión explosivos Río Tinto S.A. 2

Abonos Sevilla, S.A.

Aceites y Proteínas, S.A. (ACEPROSA)

Aceros Especiales S.A.

Aiscondel, S.A.

Alcudia

Altos Hornos de Vizcaya

Altos Hornos ingenieros Consultores, S.A.

(AHINCO)

Aluminio de Galicia

Antracitas de Fabero, S.A.

Arlesa, aceiteras reunidas de Levante, S.A.

Astilleros del Cadagua

Astilleros y Talleres del Noroeste (ASTANO)

Automóviles de Turismos Hispano-Ingleses S.A.

(AUTHI)

Auxini Ingeniería Española, S.A (AUXIESA)

Babcock \& Wilcox, S.A.

Banús Spada, S.A.

Barreiros Diesel, S.A.

Basf Española S.A.

Bayer Hispania Industrial, S.A.

Bazán
Bendibérica, S.A. 1

Bostik, S.A. 1

Brown and Root Española, S.A. 3

1 Brown and Root Española, S.A.;

1 Construcciones Sala Amateu 1

1 C.E.C. Ibérica 1

1 Campo Ebro Industrial, S.A. 2

6 Carbonoil Ibérica, S.A. 1

Casa Buades, S.A. 1

Cementos del Mar, S.A. 2

Cementos del Mediterráneo, S.A. 1

Cementos Molins, S.A. 1

Cementos Rezola, S.A. 3

Central Corsetera 1

Centrales Nucleares del Norte S.A.

(NUCLENOR) 2

Ceplástica 1

CEPSA 11

Cerámica Gaya-Marazzi, S.A. 2

Cía Nacional del Oxígeno 1

Ciba Geigy, S.A. 1

Colorificio 2

Compañía Auxiliar de Ferrocarriles, S.A. (CAF) 1

3 Compañía Española de Minas Río tinto, S.A. 2 


\begin{tabular}{|c|c|c|c|}
\hline $\begin{array}{l}\text { Nombre de } \\
\text { la empresa } \\
\text { licenciada }\end{array}$ & $\begin{array}{l}\text { Número } \\
\text { de } \\
\text { contratos }\end{array}$ & $\begin{array}{l}\text { Nombre de } \\
\text { la empresa } \\
\text { licenciada }\end{array}$ & $\begin{array}{c}\text { Número } \\
\text { de } \\
\text { contratos }\end{array}$ \\
\hline Compañía Española de Penicilina y & & Fosfórico Español, S.A. & 1 \\
\hline Antibióticos S.A. (CEPA) & 2 & FW Iberia, S.A. & 13 \\
\hline Compañía General de Sondeos, S.A. & 2 & Gallina Blanca, S.A. & 1 \\
\hline Compañía Ibérica Gea, S.A. & 1 & Gas Natural S.A. (GENESA) & 6 \\
\hline Continental Oil Company of Spain (CONOCO) & 1 & General de Confecciones, S.A. & 1 \\
\hline Cristalería Española, S.A. & 7 & Hidroeléctrica del Cantábrico, S.A. & 2 \\
\hline Derivados del Azufre, S.A. (DASA) & 7 & Hispanica de Petróleos, S.A. (HISPANOIL) & 3 \\
\hline Dow Unquinesa, S.A. & 3 & Huarte y Compañía, S.A. & 1 \\
\hline Dragados y Construcciones, S.A. & 11 & HUNOSA & 8 \\
\hline Duro-Felguera. S.A. & 4 & Industrias Químicas Asociadas, S.A. & 2 \\
\hline Duro-Felguera, S.A; Fábrica de Mieres, S.A.; & & La Maquinista terrestre y Marítima, S.A. & 3 \\
\hline Sociedad Hullera Española, S.A.;Sociedad & & La Papelera Española, S.A. & 1 \\
\hline Industrial Asturiana Santa Bárbara, S.A. & 1 & Laboratorios Almirall, S.A. & 3 \\
\hline Ebro- Compañía de Azucares y Alcoholes, S.A. & 6 & Lummus Española S.A. (LESA) & 1 \\
\hline Electricidad Goyarola-Díez Galvez S.A. & 1 & Luso Española de Porcelanas, S.A. & 1 \\
\hline Electroquímica Andaluza & 1 & Manufacturas de Corcho Armstrong, S.A. & 1 \\
\hline Electroquímica de Flix, S.A. & 3 & Manufacturas Metálicas Madrileñas & 1 \\
\hline Empresa Nacional Siderúrgica (ENSIDESA) & 37 & Marconi Española, S.A. & 1 \\
\hline ENASA & 4 & Mataderos Frigoríficos Españoles, S.A. & \\
\hline Empresa Nacional Calvo Sotelo (ENCASO) & 1 & (MAFRIESA) & 1 \\
\hline ENDASA & 3 & Maquinaria Textil del Norte de España & \\
\hline ENDESA & 3 & (MATESA) & 2 \\
\hline Energía e Industrias Aragonesas, S.A. & 1 & Metalurgia y Ferroaleaciones Especiales, S.A & \\
\hline Española del zinc S.A. & 1 & (MEFESA) & 1 \\
\hline Esso exploration Spain Inc. & 1 & Minas de Almagrera, S.A. & 1 \\
\hline Esso Petróleos Españoles, S.A. & 4 & Motor Ibérica, S.A. & 4 \\
\hline Estudios y Proyectos Técnicos S.A. (EPTYSA) & 1 & Nespral y Compañía, S.A. & 1 \\
\hline Etileno, S.A. & 1 & Nitrogas, S.A. & 3 \\
\hline Fábrica española de productos químicos & & Nueva Montaña Quijano, S.A. & 1 \\
\hline y farmacéuticos, S.A. (FAES) & 1 & Odiel Química, S.A. & 1 \\
\hline FEDISA, ingenieros Navales, S.A. & 1 & Osram, S.A. & 1 \\
\hline Fabricación Española de Fibras & & Papelera del Centro, S.A. & 1 \\
\hline Textiles Artificiales, S.A. (FEFASA) & 2 & Papelera Española, S.A. & 2 \\
\hline Fábrica Española de Magnetos, S.A. (FEMSA) & 2 & Papeleras Reunidas, S.A. & 2 \\
\hline Fibras Esso & 16 & Patricio Echeverría, S.A. & 1 \\
\hline Fibras Minerales, S.A. & 3 & Perfil del Frío, S.A. & 1 \\
\hline Foret, S.A. & 2 & Petroliber & 1 \\
\hline
\end{tabular}




\section{APÉNDICE (continuación)}

\begin{tabular}{|c|c|c|}
\hline $\begin{array}{l}\text { Nombre de } \\
\text { la empresa } \\
\text { licenciada }\end{array}$ & $\begin{array}{c}\text { Número } \\
\text { de } \\
\text { contratos }\end{array}$ & $\begin{array}{l}\text { Nombre de } \\
\text { la empresa } \\
\text { licenciada }\end{array}$ \\
\hline
\end{tabular}

Petroquímica Española, S.A. (PETRESA)

Philip Morris España, S.A.

Piher Semiconductores, S.A.

Pirelli, S.A.

Portland de Mallorca, S.A.

Potasas de Navarra, S.A.

Procon Ibérica, S.A.

Productos Químicos Esso, S.A.

Pryca, S.A.

Pulcra, S.A.

Pulptex Iberia, S.A.

R.Oyarsun y Cía, S.A.

Refinería de Petróleos de Escombreras, S.A.

(PETRESA)

RENFE

REPOSA

Revestimientos Cerámicos S.A. (RECESA)

Río Gulf Petrolquímica, S.A.

Río Tinto Patiño, S.A.

\section{S.A. Basconia}

S.A. Echevarría

S.A. La Veneciana

S.A. Echevarría

S.E. del Acumulador Tudor

Saltos del Sil, S.A.

SAVA, Modificaciones y Transformaciones, S.A. (MOTRANSA)

SETIM Ibérica

Shell España N.V.

Siemens Industria Eléctrica, S.A.

Snam Auxini Proyectos, S.A

Sociedad Española del Oxígeno, S.A.(S.E.O)

Sociedad Bilbaína de Maderas y

Alquitranes, S.A.
5 Sociedad de Proyectos para Obras

1 Técnicas Peninsulares (OTPSA)

2 Sociedad de Trabajos y Obras

1 Marítimas S.A. (SOTOMAR)

2 Sociedad Española de Carburos

6 Metálicos, S.A.

1 Sociedad española de Construcciones

5 Electromecánicas, S.A. (SECEM) 1

1 Sociedad Española del Oxígeno, S.A. 1

1 Sociedad Minera y Metalúrgica de

1 Peñarroya, S.A. 3

1 Stein\&Roubaix Española, S.A. 6

Talleres Kaeser, S.A. 1

1 Talleres Santa Bárbara, S.A. 1

1 TAMOIN 1

5 Tasada y Beltrán, S.A. 2

1 Técnicas Siderúrgicas, S.A. (TS) 1

2 Tenneco España Inc. 7

3 Termicas Asturianas 1

2 Textiles del Sur, S.A. 2

4 Textiles y Bordados, S.A. 1

1 Transportes de Petróleos, S.A. 1

1 Tubacex-Compañía Española de

2 Tubos por Extrusión, S.A. 3

2 UNINSA 9

Unión Carbide Navarra, S.A. 3

1 Union Eléctrica Canarias, S.A. 5

1 Unión Explosivos Río Tinto, S.A. 5

3 Unión Naval de Levante, S.A. 5

1 Uralita, S.A. 5

3 Victorio Luzuriaga, S.A.

1 Vidriera Vilella, S.A. 2 